\title{
Autoerythrocyte Sensitization Syndrome Presenting with General Neurodermatitis: Factitious Purpura or Psychophysiological Entity?
}

Hengguang Zhao $\cdot$ Fuling Luo $\cdot$ Hui Li

To view enhanced content go to www.dermtherapy-open.com Received: February 20, 2012 / Published online: April 17, 2012

(C) The Author(s) 2012. This article is published with open access at Springerlink.com

\section{ABSTRACT}

Autoerythrocyte sensitization syndrome (AES) is a disorder characterized by recurrent, spontaneous, painful bruising in patients with underlying psychosis and neurosis. Its pathogenesis is uncertain. Purpuric lesions reproduced by injections of washed autologous erythrocytes suggest an autoimmune etiology. The authors described a 50-year-old woman who presented with recurrent episodes of painful bruising for 8 months, along with a 2-year history of general chronic neurodermatitis. A diagnosis of AES was made

H. Zhao · H. Li (ه)

Department of Demoto-Venereology, The First Affiliated Hospital of Chongqing Medical

University, Chongqing 40016, China

e-mail: lihui131128@vip.163.com

F. Luo

Pharmaceutical Preparation Section, The First Affiliated Hospital of Chongqing Medical

University, Chongqing 40016, China

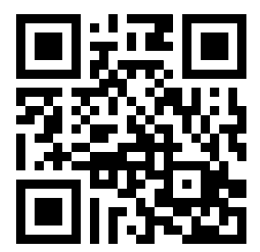

Enhanced content for this article is available on the journal web site: www.dermtherapy-open.com on the basis of the clinical symptoms and laboratory examinations results: the positive autoerythrocyte sensitization test and psychophysiologic derangement. However, the noticeable symptoms, simultaneously identical distribution of the ecchymosis and neurodermatitis lesions, as well as concomitant symptom of itching over the ecchymosis, created doubts regarding the real etiopathogenesis of the ecchymosis and whether the ecchymosis in the present patient were just factitious purpura. The authors discuss the rarity of this case, which to the authors' knowledge, had not been previously reported in the literature.

Keywords: Autoerythrocyte sensitization syndrome; Bruising; Ecchymosis; Lesions; Neurodermatitis; Psychogenic purpura

\section{INTRODUCTION}

Autoerythrocyte sensitization syndrome (AES) was first described by Gardner and Diamond [1] in 1955, when four women with painful bruising were depicted. Patients with AES 
typically present with the development of recurrent, spontaneous, painful ecchymosis, frequently preceded by a prodrome of pain or itching of the skin. Over 100 cases have been reported since then [2], and nearly all cases were in women $[3,4]$. The patients are sensitive to their own red blood cells injected intradermally, and underlying coagulopathies are thought to be absent. Psychophysiologic complaints are so frequent that AES has also been referred to as psychogenic purpura. Here, the authors described a 50-year-old woman with AES with a history of general chronic neurodermatitis recently studied in the authors' institution, who was subjected to extensive investigations over a period of 6 months before the final diagnosis.

\section{CASE REPORT}

The 50-year-old woman had recurrent episodes of painful bruising over the neck, thorax, abdomen, lumbus, and the four extremities for 8 months, whilst the scalp, face, nucha, back, and buttocks were not affected (Fig. 1). The patient complained that the bruises appeared spontaneously and were not associated with any inducement, but were accompanied by a gradual increase in pain for approximately 2 days. The patient had a 2-year history of general chronic neurodermatitis without any bleeding manifestations, such as melena, hematuria, and menorrhagia. The neurodermatitis had usually recurred approximately every 4-6 months with severe itching over the lesions, and the last relapse was approximately 3 months prior to the study. Symptoms of itching could be relieved by oral antihistamines or corticosteroids; however, the ecchymosis was unaffected. Nonetheless, the patient did not frequently use topical or systemic corticosteroids for fear of potential side effects. A detailed inquiry revealed that prick-acupuncture management was usually performed by the patient to relieve the pruritus since 9 months prior to the study when the penultimate episode of neurodermatitis initially broke out.

Physical examination at the time of bruising revealed scattered ecchymosis with lesions varying in size from 0.5 to $10.0 \mathrm{~cm}$. The lesions were tender, without bleeding, ulceration, or scarring. Dispersive, noticeable excoriation could be seen on the same regions as the ecchymosis lesions of the neck, thorax, abdomen, lumbus, and the four extremities, whilst the scalp, face, nucha, back, and buttocks were not affected by either. A detailed hematologic evaluation showed no coagulation factor deficiencies. The amount of erythrocytes, leukocytes, hemoglobin, and platelets in a blood routine examination were normal. Extensive serum chemical analyses showed all of the following indexes were negative: platelet function, von Willebrand factor, blood clotting, antibodies against platelets, erythrocyte sedimentary rate, rheumatoid factor, antinuclear antibodies, anti-dsDNA, proliferating cell nuclear antigen (PCNA), anticardiolipin antibodies, Treponema pallidum particle agglutination (TPPA), and anti-HIV. Tests of tuberculin-purified protein derivative on the right forearm and a capillary fragility experiment over both forearms also remained negative. No clinical gastrointestinal or genitourinary hemorrhaging was evident by the occult blood test in feces and urine, and no organic diseases were revealed by a urine routine examination, chest X-ray, and type-B ultrasonic examination.

An intradermal test for autoerythrocyte sensitization was performed, with $0.1 \mathrm{~mL}$ of the patient's washed red blood cells injected in 


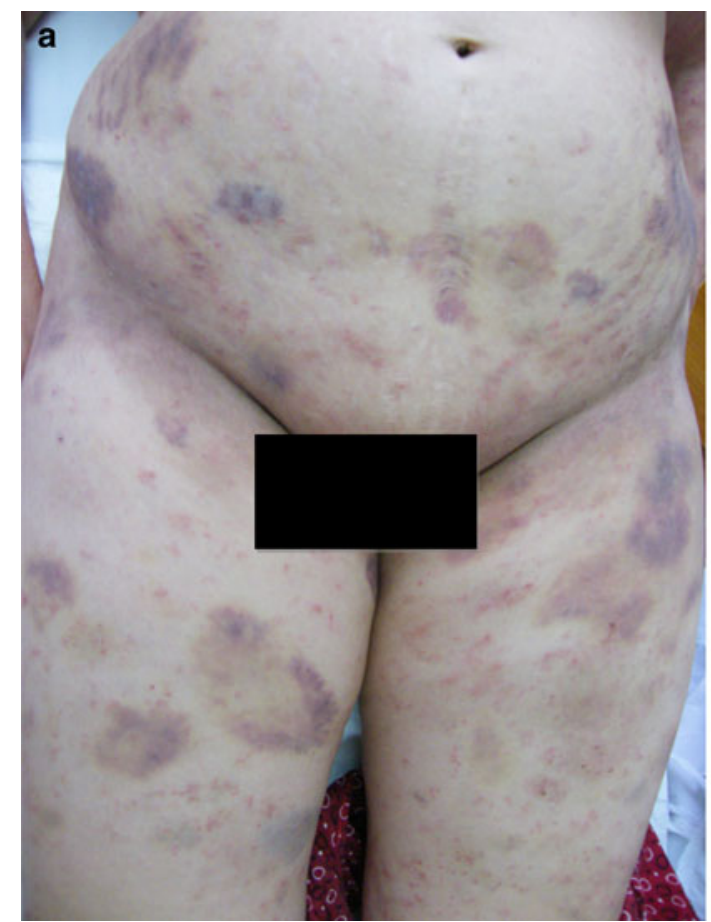

Fig. 1 A 50-year-old woman with recurrent ecchymosis over the neck, thorax, abdomen, lumbus, and the four extremities (a), while the scalp, face, nucha, back, and

the left forearm, and a saline control on the opposite side. The injection areas were overlaid with sterile carbasus to avoid any disturbance. An immediate burning sensation appeared on the test site. Approximately $24 \mathrm{~h}$ later, the patient developed a painful ecchymotic reaction on the left forearm, similar to the spontaneous lesions, whilst no reaction was observed on the control side (Fig. 2). Skin biopsies of hemorrhage lesions were performed on the right forearm and the left abdominal wall, the former showed a slight perivascular inflammatory infiltration (Fig. 3), whilst the latter showed intense erythrocytes extravasation or hemorrhage in the dermis and subcutis (Fig. 4).

Apart from the general chronic neurodermatitis, the patient had no other important dermopathic history. However, the patient complained of multiple stressors in

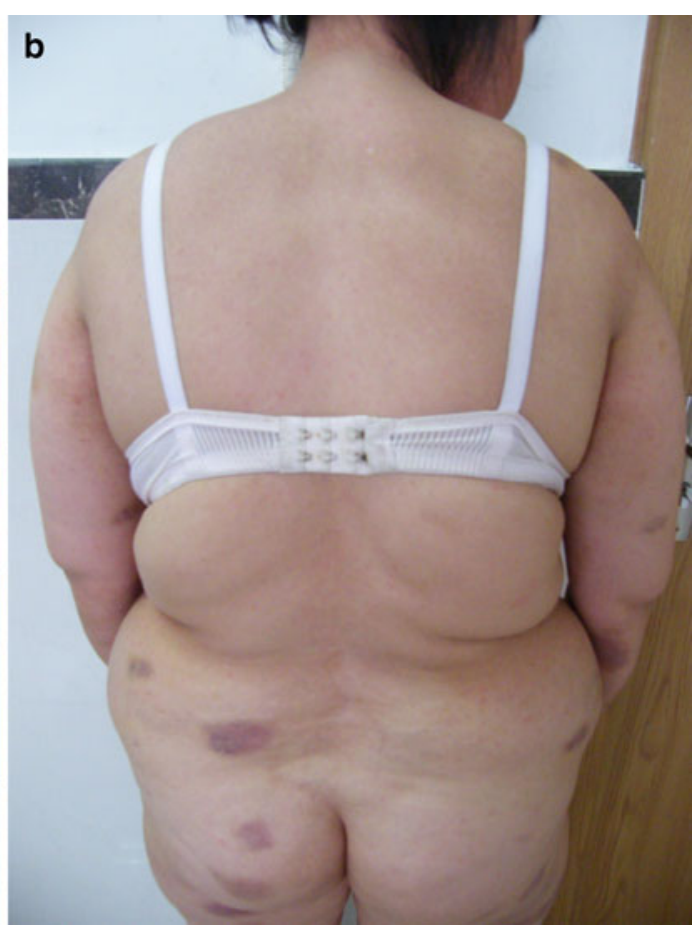

buttocks were unaffected (b). Erythra of chronic neurodermatitis can also been seen following the same distribution


Fig. 2 The test for AES on the left forearm with painful ecchymosis within $24 \mathrm{~h}$ after injection (a), and the negative result of a saline control on the opposite side (b)

daily life, and anxiety and depression often disturbed her. Furthermore, the motive for suicide emerged occasionally. A high score of 




Fig. 3 Biopsy of right forearm showed a slight perivascular inflammatory infiltrate (original magnification $\times 40$ )



Fig. 4 Biopsy of left abdominal wall revealed an intense erythrocytes extravasation or hemorrhage in the dermis and subcutis (original magnification $\times 40$ )

falsehood was revealed by psychiatric examinations using the Minnesota Multiphasic Personality Inventory. The patient was empirically given oral antihistamines and anxiolytics, and stopped acupuncture. The patient also began family therapy, environmental manipulation, and relaxation measures. The ecchymosis reduced and finally diminished approximately 4 weeks later, with a follow-up of stationary phase for more than 18 months.

\section{DISCUSSION}

The pathogenesis of AES remains unknown. Groch et al. [5] tried to identify a single biochemical agent that could be responsible for the characteristic skin lesions. Groch et al. proposed phosphatidylserine, which could be isolated from the erythrocyte membrane, to be the substance causing the disease. However, patients who were injected with phosphatidylserine showed no reaction [6]. Thereafter, most investigators proposed psychosomatic components to be one of the important contributors eventually leading to the outbreak of the disease [7]. Agle and Ratnoff [8] suggested that AES might be a psychophysiological entity, in which the reactions to psychosomatic instabilities involve the autonomic nervous system, and is a way of relieving anxiety. Such psychosomatic instabilities include emotional stress, hysteria, and masochism, which tended to erupt and become more severe during the course of the disease, while antipsychotic drugs, antidepressants, or psychotherapy were often helpful. Agle and Ratnoff $[9,10]$ believed that these patients always presented with hysterical and masochistic traits, depression, anxiety, and the inability to deal appropriately with their own hostile feelings, and hypothesized that psychogenic purpura appearing in the context of emotional stress was an interesting manifestation of a conversion reaction. Jacobi [11], who had questioned why certain people reacted with vascular symptoms instead of more common conversion symptoms under certain stress situations, stated that specific psychological mechanisms could influence the central nervous system, and this mechanism might be expressed as bleeding episodes or stigmata. Unfortunately, no more details have been revealed. 
Nowadays, the original theory proposed by Gardner and Diamond [1] is no longer widely accepted. Apart from the hypothesis of autoimmunity and psychophysiology, some investigators still highlight the mechanical injury of the skin. These investigators proposed that the mild trauma to the skin could at least be partially responsible for the onset of ecchymosis. A case of AES was reported by Levin et al. [12] as early as 1969. In this case, a teenage girl presented with recurrent, painful ecchymosis and autoerythrocyte sensitization was finally proved to be factitious purpura by means of plaster casts. Hersle and Mobacken [13] also demonstrated another case in which an intra-uterine copper device caused marked exacerbation of the ecchymosis.

Additional cases also showed the onset of autoerythrocyte sensitization could be preceded by surgical procedures or other forms of trauma [14]. Analogously, the correlation between the ecchymosis and acupuncture in the present patient created doubts with regards to a diagnosis of AES. Firstly, the ecchymosis was almost identically distributed over the neck, thorax, abdomen, lumbus, and extremities, where the prick-acupuncture management had been performed, whilst the other sites were not affected. This suggested the possibility that the ecchymosis were just factitious purpura resulting from the mechanical injury of the prick-acupuncture. This coincides with the fact that it was more convenient to perform the acupuncture on the regions of the neck, thorax, abdomen, lumbus, and extremities than the buttocks, back, and scalp. Furthermore, the autoerythrocyte sensitivity autoimmunity reaction should arouse the lesions symmetrically and uniformly over the body. Secondly, the coincidental timing seems to be more persuasive: the ecchymosis occurred directly following the administration of acupuncture and disappeared gradually after the acupuncture was stopped.

According to Sawhney and Arora [15], in factitious purpura, there were usually no "premonitions" before the onset of the lesions. The results of skin sensitivity tests tended to follow the patient's perception of their doctor's expectations, and were positive only if the injected areas were accessible to them. In view of the present patient, the sensation of intermittent mild itching was definite, but whether it was actually a neurodermatitis symptom or one of the premonitions of ecchymosis is unknown. Furthermore, in the present patient, itching was more prevalent than pain. Moreover, the patient was blinded to the purpose of the injections, and the injection areas were overlaid by sterile carbasus to avoid access. The close observation following intradermal injections confirmed that the patient had not interfered with the dressings. As a result, it was still difficult to differentiate AES from the factitious purpura, especially in the absence of hematological abnormalities.

In conclusion, one of the reasonable interpretations for the present patient probably lies on the hypothesis of synergism between psychoimmunology and mechanical injury of the skin. As for the authors' inference, the psychosomatic instabilities might have initially disturbed the skin's immunological function and weakened the skin's blood capillaries, so that even a mild trauma of the skin would damage the capillary walls and eventually result in the permeation of red corpuscles.

\section{ACKNOWLEDGMENTS}

This study was supported by the Sanitary Science Foundation of Chongqing 
Municipality, China (Grant No. 2010-2-014). H.Z. is the guarantor for this article, and takes responsibility for the integrity of the work as a whole.

Conflict of interest. The authors declared that they had no conflicts of interests.

Open Access. This article is distributed under the terms of the Creative Commons Attribution Noncommercial License which permits any noncommercial use, distribution, and reproduction in any medium, provided the original author(s) and source are credited.

\section{REFERENCES}

1. Gardner FH, Diamond LK. Autoerythrocyte sensitization. A form of purpura producing painful bruising following autosensitization to red blood cells in certain women. Blood. 1955;10:675-8.

2. Karatosun V, Satoğlu S, Gŭnal İ, et al. Autoerythrocyte sensitization (Gardner-Diamond) syndrome mimicking compartment syndrome. Arch Orthop Trauma Surg. 2003;123:370-1.

3. Kalla G, Roy R, Purohit DR, et al. Painful bruising syndrome. Indian J Dermatol. 2001;46:118-9.

4. Chatterjee M, Jaiswal AK. Painful bruising syndrome. Indian J Dermatol Venereol Leprol. 2002;68:347-8.

5. Groch GS, Finch SC, Royoway W, et al. Studies in the pathogenesis of autoerythrocyte sensitization syndrome. Blood. 1966;28:19-33.
6. Kirschner MH, Hofmann GO, Markewitz A, et al. Osteosynthetic reconstruction in a patient with Gardner-Diamond syndrome. J Trauma. 1995;38: 392-5.

7. Yasmin A, Soutar R, McKay P, et al. Autoerythrocyte sensitisation syndrome clinical-an unusual cause of bruising. J R Coll Physicians Edinb. 2008;38:311-3.

8. Agle DP, Ratnoff OD. Purpura as a psychosomatic entity. Arch Intern Med. 1962;109:89-98.

9. Ratnoff OD, Agle DP. Psychogenic purpura: a re-evaluation of the syndrome of autoerythrocyte sensitization. Medicine. 1968;47:475-500.

10. Agle DP, Ratnoff OD. Conversion reactions in autoerythrocyte sensitization. Arch Gen Psychiatry. 1969;20:438-47.

11. Jacobi E. Psychogene Spontanblutungen der Haut. Aus der psychiatrischen und Nervenklinik der Universität Königsberg. 1929;88:631-45.

12. Levin RM, Chodosh R, Sherman JD. Factitious purpura simulating autoerythrocyte sensitization. Ann Intern Med. 1969;70:1201-6.

13. Hersle K, Mobacken H. Autoerythrocyte sensitization syndrome (painful bruising syndrome). Report of two cases and review of the literature. Br J Dermatol. 1969;81:574-87.

14. Vun YY, Muir J. Periodic painful purpura: fact or factitious? Australas J Dermatol. 2004;45:58-63.

15. Sawhney MPS, Arora G, Arora S, et al. Undiagnosed purpura: a case of autoerythrocyte sensitization syndrome associated with dermatitis artefacta and pseudoainhum. Indian J Dermatol Venereol Leprol. 2006;72:379-81. 